\title{
Enhanced Quantification of CFP:YFP Intramolecular FRET Using Multiphoton Excitation Microscopy
}

\author{
Mark A. Rizzo* and David W. Piston* \\ * Department of Molecular Physiology and Biophysics, 735 Light Hall, Vanderbilt University, \\ Nashville, TN 37232
}

A popular strategy for generating genetically encoded biosensors utilizes fluorescence resonance energy transfer (FRET) between cyan (CFP) and yellow (YFP) fluorescent proteins [1]. These probes generally consist of a conformationally sensitive biosensing protein construct flanked by CFP and YFP [2]. Typically, a maximum two-fold change in the CFP:YFP FRET ratio occurs in response to interaction of the biosensor domain with its agonist. The use of this FRET pairing is hampered by both a poor dynamic range and the broad overlap between CFP and YFP excitation spectra (FIG 1). Thus obtaining both accurate quantification of the FRET ratio along with good spatial and temporal resolution is a challenging problem in the context of live cell imaging. Here we used tandem YFP:CFP constructs targeted to different cellular compartments in order to assess the accuracy of FRET quantification along with temporal and spatial resolution for different live cell imaging methods. A filter-based wide-field microscopy approach allowed good spectral separation and thus adequate quantification of FRET, although the requirement for filter switching between channels limited the spatial and temporal resolution of highly dynamic systems. Laser scanning confocal microscopy with an Ar laser excitation source $(458 \mathrm{~nm})$ and two collection channels for CFP and YFP improved the temporal and spatial resolution by allowing simultaneous collection of CFP and YFP fluorescence. However, this method suffered from poor quantification of FRET due to crosstalk excitation of YFP. Laser scanning multiphoton excitation microscopy using a tunable TiSaph laser source enabled selection of an optimal excitation wavelength for quantitative FRET imaging and simultaneous collection of CFP and YFP emission using two channels. FRET images of a cell expressing a YFP:CFP fusion protein obtained using 458nm excitation and multiphoton excitation are compared in FIG 2. The FRET ratio obtained using 458nm excitation varied extensively within the cell, especially near the thin cellular edges. In contrast, the FRET image obtained using multiphoton excitation had a more uniform ratio throughout the cell. In conclusion, elimination of excitation crosstalk using multiphoton excitation resulted in consistent FRET quantification along with good spatial and temporal resolution.

References

[1] G. Patterson, D. W. Piston, and B.G. Barisas Anal Biochem. 284 (2000) 438.

[2] J. Zhang et al., Nat. Rev. Mol. Cell Biol. 3 (2002) 906.

[3] Funding for this work was provided by NIH grants DK60275 (M. A. R.), and DK53434 and

CA86283 (both to D. W. P.), and by NSF grant BBI-9871063 (to D. W. P.). 


\section{Excitation Spectra}

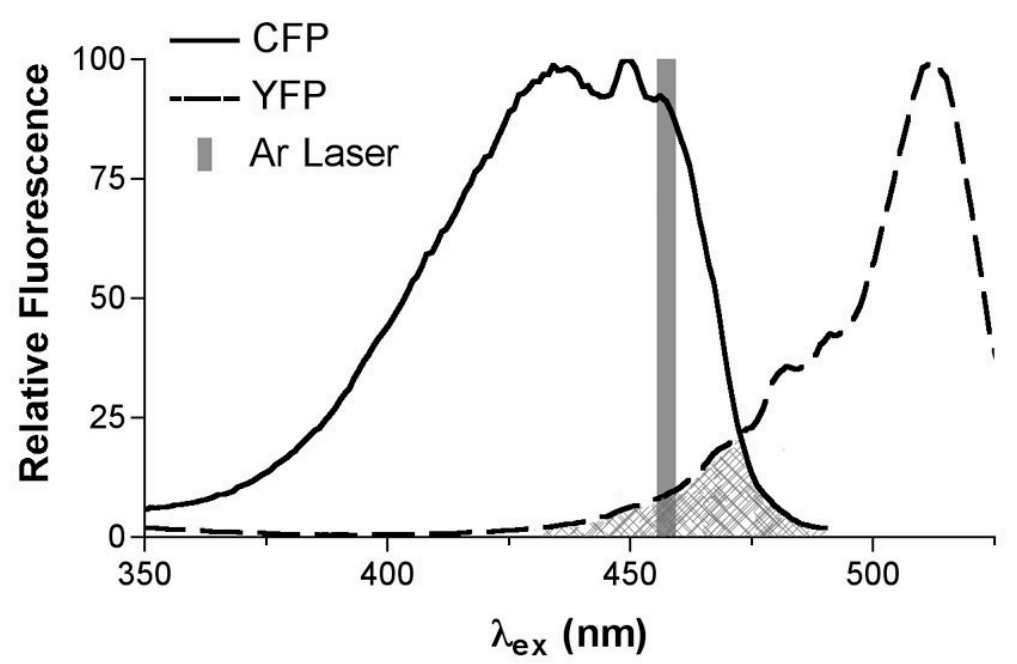

FIG 1. Spectral crosstalk between CFP and YFP excitation spectra. Excitation spectra of affinity purified CFP and YFP were measured in a fluorometer. Overlap of the spectra is indicated by the hatched area. The $457 \mathrm{~nm}$ Ar laser line used for CFP excitation in confocal microscopy is depicted.
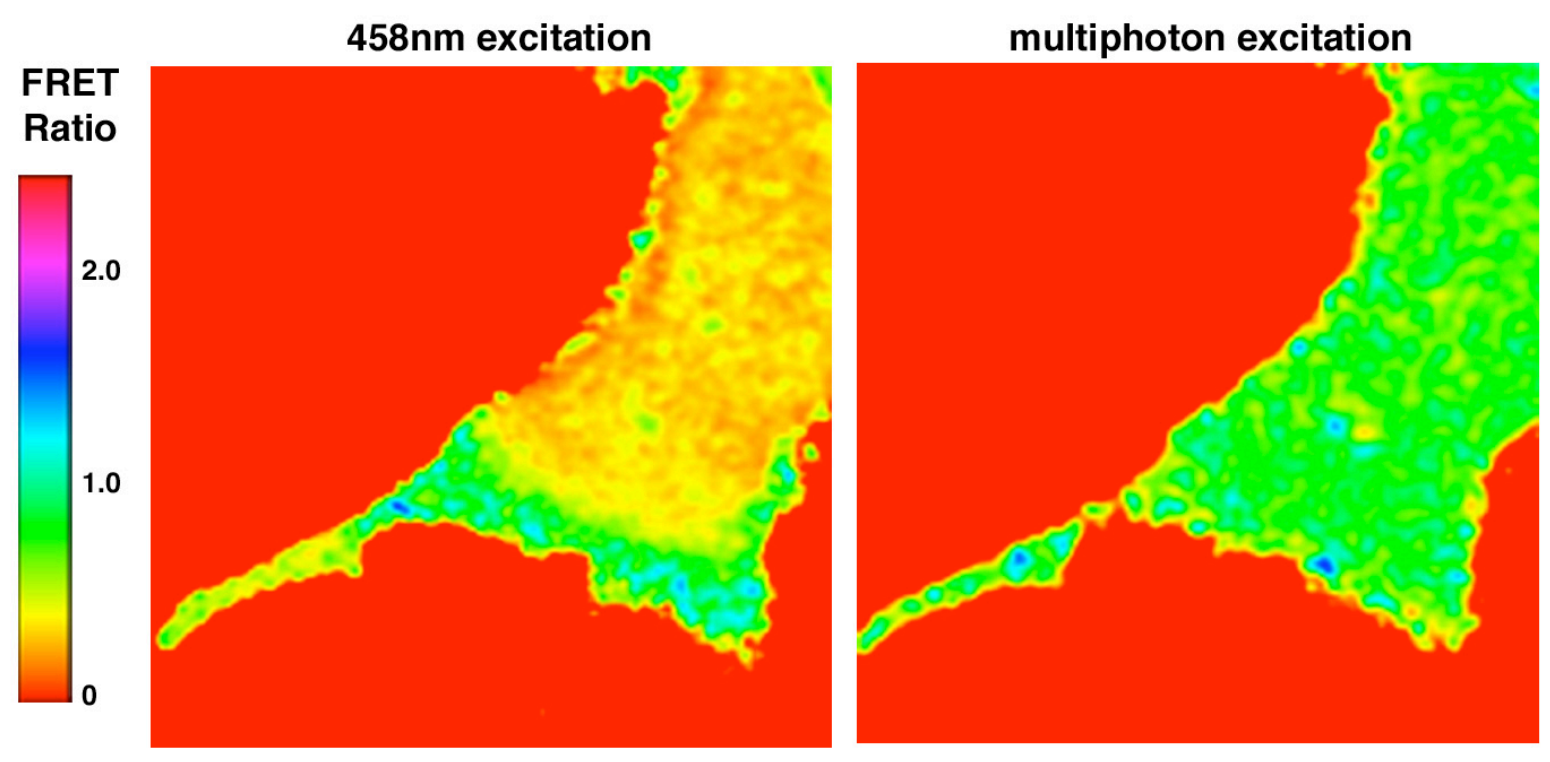

FIG 2. FRET images of a COS-7 cell expressing a YFP:CFP fusion protein were taken using confocal microscopy (458 excitation, left) and with multiphoton excitation (right) using equivalent detection conditions. A FRET ratio image was generated from separate YFP and CFP images using NIH Image software. 